DIGITALCOMMONS @WAYNESTATE-
Clinical Research in Practice: The Journal of Team Hippocrates

\title{
Addition of an anti-staphylococcal beta lactam to standard antibiotic therapy does not decrease 90 -day mortality of MRSA bacteremia versus standard therapy alone
}

Monica Hessler

Wayne State University School of Medicine, gf4909@wayne.edu

Follow this and additional works at: https://digitalcommons.wayne.edu/crp

Part of the Medical Education Commons, and the Translational Medical Research Commons

\section{Recommended Citation}

HESSLER M. Addition of an anti-staphylococcal beta lactam to standard antibiotic therapy does not decrease 90 -day mortality of MRSA bacteremia versus standard therapy alone. Clin. Res. Prac. Oct 9 2020;6(2):eP2351. https://doi.org/10.22237/crp/1593561720

This Clinical Decision Report is brought to you for free and open access by the Open Access Journals at DigitalCommons@WayneState. It has been accepted for inclusion in Clinical Research in Practice: The Journal of Team Hippocrates by an authorized editor of DigitalCommons@WayneState. 


\title{
Addition of an anti-staphylococcal beta lactam to standard antibiotic therapy does not decrease 90-day mortality of MRSA bacteremia versus standard therapy alone
}

MONICA HESSLER, Wayne State University School of Medicine, gf4909@wayne.edu

\begin{abstract}
A clinical decision report appraising Tong SYC, Lye DC, Yahav D, et al. Effect of vancomycin or daptomycin with vs without an antistaphylococcal $\beta$-lactam on mortality, bacteremia, relapse, or treatment failure in patients with MRSA bacteremia: a randomized clinical trial. JAMA. 2020;323(6):527-537. https://doi.org/10.1001/jama.2020.0103 for a patient with multiple comorbidities including heart failure, severe aortic regurgitation, aortic aneurysm and intellectual disability being treated for MRSA bacteremia who is intubated in the ICU.
\end{abstract}

Keywords: $\quad$ MRSA, beta lactam, cephalosporin, vancomycin, anti-staphylococcal, bacteremia

\section{Clinical Context}

Dominic Edwards (Pseudonym) is a 34-year-old man with developmental delay and a history of heart failure with reduced ejection fraction (EF of 30\%), recurrent pleural effusions, $7.4 \mathrm{~cm}$ ascending aortic aneurysm, severe aortic regurgitation, and restrictive lung disease secondary to severe scoliosis, with nighttime BiPAP dependency. He was brought to the ED by his mother, his primary caregiver, after she thought he "wasn't doing well and was drowsy." He had struggled to use his BiPAP for the last two nights. He was found to be severely acidotic and was subsequently intubated in the MICU.

On hospital day five, Mr. Edwards spiked a fever of $38.1^{\circ} \mathrm{C}$. Blood cultures grew methicillin resistant Staphylococcal aureus (MRSA), and respiratory culture showed MRSA and Pseudomonas aeruginosa. The Infectious Disease service was consulted. At bedside, Mr. Edwards was intubated and diaphoretic, but still arousable and answering questions by nodding. With assistance from his mother, we discussed our plans. Mrs. Edwards stated that Dominic "just wasn't himself," and she "just wanted her son back." As she is his primary caregiver, his current hospitalization was taxing on her, as she had other children at home in her care. He had several prior hospitalizations for similar problems, which had been challenging for his mother, both emotionally and financially. She repeatedly conveyed to us how much her son meant to her, and that her main priority was to get him home soon. At this point, our patient had already started vancomycin and aztreonam for empiric coverage, and we recommended switching to cefepime for Pseudomonas coverage. Per the Infectious Disease Fellow, the combination of cefepime and vancomycin could act synergistically against MRSA, while covering Pseudomonas. Because we were unfamiliar with that clinical reasoning, we took the opportunity to learn more, as this clinical presentation is quite common in clinical practice.

MONICA HESSLER is a medical student at Wayne State University School of Medicine. 
HESSLER M. Addition of an anti-staphylococcal beta lactam to standard antibiotic therapy does not decrease 90-day mortality of MRSA bacteremia versus standard therapy alone. Clin. Res. Prac. Oct 9 2020;6(2):eP2351. https://doi.org/10.22237/crp/1593561720
Clinical Research in Practice The Journal of Team Hippocrates

VOL 6 ISS 2 / eP2351 / OCTOBER 9, 2020 https://doi.org/10.22237/crp/1593561720

\section{Clinical Question}

Is there synergistic benefit and reduced bacteremia duration if vancomycin is combined with a cephalosporin or beta lactam to treat MRSA bacteremia?

\section{Research Article}

Tong SYC, Lye DC, Yahav D, et al. Effect of vancomycin or daptomycin with vs without an antistaphylococcal $\beta$-lactam on mortality, bacteremia, relapse, or treatment failure in patients with MRSA bacteremia: a randomized clinical trial. JAMA. 2020;323(6):527-537. https://doi.org/10.1001/jama.2020.0103

\section{Related Literature}

We searched PubMed using the search terms "MRSA bacteremia treatment vancomycin, $\beta$-Lactam," resulting in 146 items. After filtering by "Clinical Trial," 11 studies remained. Of these 11, three of the studies involved both vancomycin and a beta-lactam antibiotic. One article was found through the citation matching function and two additional trials $\underline{1}, \underline{2}$ were similar articles that were included.

The CAMERA2 study was published March 31, 2013 as an open label, randomized control study comparing vancomycin or daptomycin, both with and without an anti-staphylococcal beta lactam. $\frac{3}{\text { At }}$ the time of the publication of this paper, the study was only open for enrollment, with no results.

Casapao et al. ran a retrospective multicenter cohort study at five academic or community hospitals in the United States on 93 adults with MRSA bacteremia who were treated with initiation of an intravenous beta lactam within 24 hours of initiating vancomycin as compared to standard therapy of intravenous vancomycin. The primary outcome was clinical failure which included 30-day mortality, persistent bacteremia $\geq 7$ days, bacteremia relapse, or change in antibiotic therapy due to clinical worsening. ${ }^{-1}$ At the completion of the trial, combination therapy did not decrease the rate of clinical failure but had expedited bacteremia clearance. ${ }^{-}$Given the retrospective nature of this article and small sample size, its Strength of Recommendation Taxonomy (SORT) is level 2 and was not chosen for appraisal.

Georges et al. performed a prospective crossover study, written in French, which was carried out on 20 patients with severe pneumonia or bacteremia who received either vancomycin $2 \mathrm{~g}$ per day with cefpirome or standard therapy with vancomycin $2 \mathrm{~g}$ per day. The outcome was evaluated on clinical recovery, duration of ventilation, and ICU stay. Results showed that there was no significant difference in clinical outcomes between the two groups, but the bactericidal power in the sera was better in the second group. -4 This study looked promising, but after searching multiple databases, the whole paper was not available in English.

After repeating the search, one additional study was added by McCreary et al. $\stackrel{2}{-}$ This retrospective, matched cohort study was done on patients with MRSA bacteremia in four US hospitals examining the use of daptomycin and ceftaroline (DAP-CPT) compared to the use of the standard of care (SOC). Results showed that DAP-CPT treatment was initiated later in the hospital course as compared to the SOC, which resulted in shorter duration of bacteremia in the SOC group. Given the retrospective nature of this study, this study was not selected.

Tong et al. ran an open label, randomized clinical trial on 352 hospitalized patients with MRSA bacteremia at 27 different hospitals in four countries from August 2015 to July 2018. Patients were randomized to receive intravenous vancomycin or daptomycin plus an anti-staphylococcal beta-lactam or standard therapy with either vancomycin or daptomycin. Primary endpoints of the study were 90-day composite of mortality, persistent bacteremia at day five, microbiological relapse, and treatment failure. They also had secondary outcomes including mortality at days 14, 42, and 90, bacteremia remaining at days two and five, acute kidney injury (AKI), relapse, and duration of intravenous antibiotics. - This study was chosen for appraisal based on the large sample size, recent publication, and the direct application to our patient. Per the SORT criteria, this trial has level 2 evidence, given that the study is patient oriented, was not blinded, and allocation was not concealed. The overall body of research on this topic meets strength of recommendation SORT level $B$, as currently available evidence is limited. 
HESSLER M. Addition of an anti-staphylococcal beta lactam to standard antibiotic therapy does not decrease 90-day mortality of MRSA bacteremia versus standard therapy alone. Clin. Res. Prac. Oct 9 2020;6(2):eP2351. https://doi.org/10.22237/crp/1593561720
Clinical Research in Practice The Journal of Team Hippocrates

VOL 6 ISS 2 / eP2351 / OCTOBER 9, 2020 https://doi.org/10.22237/crp/1593561720

\section{Critical Appraisal}

Tong et al. held their open label, parallel group randomized control clinical trial from August 2015 through July 2018 , with follow-up completed on October 23. 2018. Hospitalized patients fit trial inclusion criteria with a positive blood culture for MRSA, ability to be randomized within 72 hours of first positive blood culture, $\geq 18$ years old, and length of stay at least 7 days after randomization. Our patient fit these inclusion criteria. Exclusion criteria included a type 1 hypersensitivity to beta lactams, known pregnancy, expected death within 48 hours, polymicrobial bacteremia, treatment limitations precluding antibiotic use, or clinicians refusing patient involvement. This last exclusion criterion is probably the largest source of bias in the study design.

Patient randomization was performed using standard computer software, and a statistician who was uninvolved in the trial made the computer-generated randomization codes. Due to cost, neither the patients nor the clinicians were blinded to the therapy. This is also a significant source of bias inherent in open label trials. Standard therapy was either vancomycin or daptomycin, compared to a beta lactam in combination with vancomycin or daptomycin. All medications were renally dose adjusted. Recommended treatment duration was 14-42 days, and if in the trial group, the first seven days with the beta lactam. Any non-antibiotic treatment was left to clinician discretion. Primary outcomes included all-cause mortality, persistent bacteremia at study day five, microbiological relapse (defined as a positive blood culture $\geq 72$ hours after a preceding negative culture) and microbiological treatment failure with positive sterile site culture for MRSA at least 14 days after randominzation. $\frac{5}{-}$ The study was closed by the data safety monitoring board prior to the goal of 440 patients enrolled due to the concern of increased incidence of acute kidney injury (AKI) in patients receiving combination therapy. Post hoc descriptions included charting creatinine changes from baseline.

A Mann Whitney $U$ test was used for the continuous outcomes and for the primary endpoint and other categorical measures, the absolute difference in proportions were reported with 95\% confidence intervals. Twenty-six patients in the combination group did not receive at least $75 \%$ of the study beta lactam doses and didn't count in the per protocol population. All data was reported and the seven patients who were lost to follow up were considered "treatment failure." There was no significant improvement of the primary endpoint composite of mortality, relapse, or treatment failure when comparing the combination group and the standard ( $35 \%$ and $39 \%$ respectively), with a difference of $-4.2 \%(95 \% \mathrm{Cl},-14.3 \%$ to $6.0 \% ; \mathrm{P}=0.42) .5$ However, persistent bacteremia on day five showed significant decline in patients treated with combination therapy, where $11 \%$ were persistently bacteremic in the combination group versus $20 \%$ in the standard (difference $=-8.9 \%, 95 \% \mathrm{Cl},-16.6$ to $-1.2 \%) . \underline{5}$

Treatment length was left to the discretion of the clinician, which could confound any differences seen in results. If patients had similar clinical pictures, but one patient had a longer duration of treatment, this would increase the likelihood that the patient with longer treatment would have bacteremia clearance. For reference, our patient was scheduled to receive 28 days of therapy due to his aortic root dilatation but received eight days of therapy with cefepime. Additionally, these study patients came from four different countries and 27 different hospitals, in which antibiotic susceptibilities differ; our local minimum inhibitory concentrations (MICs) and susceptibilities likely vary from the countries in this article. Given the incidence of AKI in the combination group with flucloxacillin and cloxacillin, more than with cefazolin, further analysis with other cephalosporins is also warranted, as it was previously found that anti-staphylococcal penicillins are associated with AKI more than cefazolin ( $12 \%$ vs $3.4 \%$ respectively). $\underline{6}$ With the early study closure, researchers were unable to achieve the primary goal of 440 patients involved in the study, resulting in fewer patients for data analysis. Finally, numerous authors released their conflicts of interest and grants/funding received from pharmaceutical companies, however no pharmaceutical brand names were used in the trial, mitigating potential funding bias.

\section{Clinical Application}

After discussion with his mother, she agreed to our combination therapy treatment recommendation. She noted that she wanted all interventions for life prolongation except surgical intervention, and her goal for her son was to get him back home under her care. Given that his respiratory sample showed both Pseudomonas aeruginosa and MRSA, the addition of cefepime was thought to serve dual purposes of treating his Pseudomonal lung infection and acting synergistically in his MRSA bacteremia. The possibility of clearing his bacteremia sooner, rooted in evidence from Tong et al.'s study, was a major driver in our treatment recommendation for our patient. In hindsight, the lack of benefit for the primary endpoint means that the answer to our Clinical Question was "no", there is not synergistic benefit of a cephalosporin administered with vancomycin for MSRA. We used a secondary endpoint in a weakly designed clinical trial as the basis of our recommendation. 
HESSLER M. Addition of an anti-staphylococcal beta lactam to standard antibiotic therapy does not decrease 90-day mortality of MRSA bacteremia versus standard therapy alone. Clin. Res. Prac. Oct 9 2020;6(2):eP2351. https://doi.org/10.22237/crp/1593561720
Clinical Research in Practice The Journal of Team Hippocrates

VOL 6 ISS 2 / eP2351 / OCTOBER 9, 2020 https://doi.org/10.22237/crp/1593561720

After discussing potential risks such as AKI, and the benefits of hastening bacteremia clearance, his mother was amenable to combination therapy. Mr. Edwards was started on combination vancomycin and cefepime on hospital day six. He received a total of eight days of combination therapy, and then continued the vancomycin for a total of 28 days due to his aortic aneurysm. With his first negative blood culture on treatment day four after the initial positive culture, we felt he had a positive response to therapy. Although the Tong et al. study showed that combination of vancomycin and a beta lactam/cephalosporin did not decrease the primary composite endpoint, Mr. Edwards cleared his bacteremia on day four of treatment, consistent with the study's secondary outcome of fewer cases of persistent bacteremia on treatment day five in the combination group (11\%) versus the standard treatment (20\%). - Unfortunately, his many comorbidities persisted, and he did not return to his baseline. Mr. Edwards remained intubated throughout his protracted hospital course but showed signs of clinical improvement from infection.

\section{New Knowledge Related to Clinical Decision Science}

With the clearance of Mr. Edwards' MRSA bacteremia on treatment day four, from our perspective this was a successful use of combination cefepime and vancomycin that correctly applied existing literature in our patient's context. However, his mother wanted a swift return to baseline so he could be discharged home, which was ultimately not met by our treatment, illustrating differences in expectations between provider and patient/family. Our optimization of antibiotics as a consulting team, rooted in available evidence, met our short-term goals for infection clearance, but we did not completely take into account the broader expectations of our patient's mother, and thus in her eyes, our treatment was still unsuccessful. Additionally, the increased risk of AKI with the possibility that this could result in dialysis dependent care would prevent Mr. Edwards' mother from achieving her desired outcome that she clearly expressed to our team. As this outcome reminds us, while meeting medical goals is something to strive for, a constant awareness of the patient's goals is required to achieve patient and family satisfaction. As medical professionals, our decisions need to be grounded in treating human beings, not diseases.

Another common topic in Clinical Decision Science is the relationship between specialist consultants and the primary treatment team. In this case, reasonable doctors can disagree. Learning how to explain a rationale for making a clinical decision is a skill we all need to foster. That would allow higher level discussions between consultants and the primary care doctors who have to integrate the evidence into the clinical management plan.

\section{References}

1. Casapao AM, Jacobs DM, Bowers DR, Beyda ND, Dilworth TJ; REACH-ID Study Group. Early administration of adjuvant $\beta$-lactam therapy in combination with vancomycin among patients with methicillin-resistant Staphylococcus aureus bloodstream infection: a retrospective, multicenter analysis. Pharmacotherapy. 2017;37(11):1347-1356. https://doi.org/10.1002/phar.2034

2. McCreary EK, Kullar R, Geriak M, et al. multicenter cohort of patients with methicillin-resistant Staphylococcus aureus bacteremia receiving daptomycin plus ceftaroline compared with other MRSA treatments. Open Forum Infect Dis. 2019;7(1):ofz538. Published 2019 Dec 31. https://doi.org/10.1093/ofid/ofz538

3. Tong SYC, Nelson J, Paterson DL, et al. CAMERA2 - combination antibiotic therapy for methicillin-resistant Staphylococcus aureus infection: study protocol for a randomised controlled trial. Trials. 2016;17(1):170. https://doi.org/10.1186/s13063-016$\underline{1295-3}$

4. Georges B, Roche $C$, Archambaud M, et al. Importance of a cefpirome-vancomycin combination on bactericidal kinetics in severe MRSA infections in intensive care. Pathologie-biologie. 2002;50(3):161-168. https://doi.org/10.1016/S0369-8114(02)00283-3

5. Tong SYC, Lye DC, Yahav D, et al. Effect of vancomycin or daptomycin with vs without an antistaphylococcal $\beta$-lactam on mortality, bacteremia, relapse, or treatment failure in patients with MRSA bacteremia: a randomized clinical trial. JAMA. 2020;323(6):527-537. https://doi.org/10.1001/jama.2020.0103

6. Weis S, Kesselmeier M, Davis JS, et al. Cefazolin versus anti-staphylococcal penicillins for the treatment of patients with Staphylococcus aureus bacteremia. Clin Microbiol Infect. 2019;25(7):818-827. https://doi.org/10.1016/i.cmi.2019.03.010 